Pontifícia Universidade Católica $_{\text {mane }}$

Carlos Andrés Aguilar Marón

Uma implementação expedita do método híbrido dos elementos de contorno para problemas de potencial e elasticidade

Tese apresentada ao Programa de Pós-graduação em Engenharia Civil do Departamento de Engenharia Civil da PUC-Rio como requisito parcial para obtenção do título de Doutor em Engenharia Civil

Orientador: Prof. Ney Augusto Dumont 
Carlos Andrés Aguilar Marón

\title{
Uma implementação expedita do método híbrido dos elementos de contorno para problemas de potencial e elasticidade
}

Tese apresentada como requisito parcial para obtenção do grau de Doutor pelo programa de Pós-graduação em Engenharia Civil do Departamento de Engenharia Civil do Centro Técnico Científico da PUC-Rio. Aprovada pela Comissão Examinadora abaixo assinadaAprovada pela Comissão Examinadora abaixo assinada.

\author{
Prof. Ney Augusto Dumont \\ Orientador \\ Departamento de Engenharia Civil - PUC-Rio \\ Prof. Raul Rosas e Silva \\ Departamento de Engenharia Civil - PUC-Rio \\ Dr. Alexandre Antonio de Oliveira Lopes \\ TECGRAF — PUC-Rio
}

Prof. José Claudio de Faria Telles

Universidade Federal do Rio de Janeiro

Prof. Rodrigo Bird Burgos

Universidade do Estado do Rio de Janeiro

Prof. Volodomyr Vasilievich Zozulya

Centro de Investigação Científica de Yucatán

Prof. José Eugenio Leal

Coordenador Setorial do Centro Técnico Científico - PUC-Rio 
Todos os direitos reservados. É proibida a reprodução total ou parcial do trabalho sem autorização da universidade, do autor e do orientador.

\section{Carlos Andrés Aguilar Marón}

Graduou-se em Engenharia Civil, pela Universidad Nacional San Antonio Abad del Cusco - Peru. Em 2006 iniciou o curso de mestrado em Engenharia Civil na PUC-Rio e titulouse em 2008, na área de estruturas, atuando na linha de pesquisa de Métodos dos Elementos de Contorno e Dinâmica das Estruturas.

Ficha Catalográfica

Aguilar Marón, Carlos A.

Uma implementação expedita do método híbrido dos elementos de contorno para problemas de potencial e elasticidade / Carlos Andrés Aguilar Marón; orientador: Ney Augusto Dumont. - Rio de Janeiro : PUC-Rio, Departamento de Engenharia Civil, 2013.

v., 87 f: il. ; $29,7 \mathrm{~cm}$

1. Tese (doutorado) - Pontifícia Universidade Católica do Rio de Janeiro, Departamento de Engenharia Civil.

Inclui referências bibliográficas.

1. Engenharia Civil - Tese. 2. Elementos híbridos de contorno;. 3. Métodos variacionais;. 4. Métodos numéricos;. 5. Elementos de contorno; . 6. Problemas de grande escala.. I. Dumont, Ney Augusto. II. Pontifícia Universidade Católica do Rio de Janeiro. Departamento de Engenharia Civil. III. Título. 


\section{Agradecimentos}

Ao Prof. Ney Augusto Dumont, pela orientação, paciência, apoio e incentivo na realização deste trabalho. Obrigado professor.

Ao $\mathrm{CNPq}$ /CAPES e à PUC-Rio, pelos auxílios concedidos, sem os quais este trabalho não poderia ter sido realizado, nem minha estada no Brasil teria sido possível.

Aos professores do departamento de Engenharia Civil da PUC-Rio.

Ao pessoal administrativo do programa de pós-graduação em engenharia civil da PUC-Rio.

Aos membros da banca, pelas diversas sugestões feitas na redação final da dissertação.

À minha família no Peru, pelo apoio incondicional em todos esses anos, meu Pai Carlos Aguilar e irmãos.

Finalmente, dedico este trabalho à memoria da minha mãe: Alejandrina. 


\section{Resumo}

Aguilar Marón, Carlos A.; Dumont, Ney Augusto. Uma implementação expedita do método híbrido dos elementos de contorno para problemas de potencial e elasticidade. Rio de Janeiro, 2013. 87p. Tese de Doutorado - Departamento de Engenharia Civil, Pontifícia Universidade Católica do Rio de Janeiro.

O desenvolvimento consistente do método convencional dos elementos de contorno (CBEM), com a adição de conceitos da versão simplificada do método híbrido dos elementos de contorno (HBEM), proveniente do potencial variacional de Hellinger-Reissner, conduz-se a um processo computacionalmente mais econômico, sem a necessidade de ter sua precisão numérica reduzida para problemas de grande escala, podendo ser bidimensional ou tridimensional, de potencial ou elasticidade. Conseguiu-se mostrar que as matrizes de potencial duplo e simples do CBEM, $\mathbf{H}$ e $\mathbf{G}$, respectivamente, cuja avaliação numérica requer a manipulação de integrais singulares e impróprias, podem ser obtidas de maneira expedita, eliminando-se quase toda a integração numérica, com exceção de algumas integrais regulares. Uma importante característica da formulação proposta, que advém da base variacional do HBEM, é a facilidade da obtenção de resultados em pontos internos, de maneira direta e sem a utilização de qualquer integral de contorno, já que a solução fundamental é a própria solução do problema. O presente trabalho pertence a um projeto cujo resultado final deve ser um código computacional para problemas de grande escala (milhões de graus de liberdade). Nesta fase, alguns exemplos numéricos foram testados para avaliar a aplicabilidade do método expedito, o seu esforço computacional e a convergência do resultado para as variáveis envolvidas no método. Para isso, foram implementados algoritmos para problemas bidimensionais de potencial e elasticidade - usando elementos lineares, quadráticos e cúbicos - e tridimensionais - usando elementos triangulares e quadrilaterais, lineares e quadráticos nos dois casos. Os códigos computacionais foram implementados focando na solução de problemas de grande escala. Espera-se que numa etapa final o projeto possa ser bem mais eficaz, com a incorporação de procedimentos do método "fast multipole".

\section{Palavras-chave}

Elementos híbridos de contorno; Métodos variacionais; Métodos numéricos; Elementos de contorno; Problemas de grande escala. 


\section{Abstract}

Aguilar Marón, Carlos A.; Dumont, Ney Augusto(advisor). An expedite implementation of the hybrid boundary element method for potential and elasticity problems. Rio de Janeiro, 2013. 87p. PhD Thesis — Departamento de Engenharia Civil, Pontifícia Universidade Católica do Rio de Janeiro.

The consistent development of the conventional boundary elements method (CBEM) by adding the concepts of the hybrid boundary element simplified method (HBEM), from the Hellinger-Reissner variational potential leads to a computationally less intensive procedure, although not necessarily less accurate for large scale, two-dimensional or three-dimensional problems of potential and elasticity. It was shown that both single-layer and double-layer potential matrices, $\mathbf{G}$ and $\mathbf{H}$, respectively, are obtained in an expeditious way that vanish almost any numerical integration, except for a few regular integrals, even $\mathbf{G}$ and $\mathbf{H}$ evaluation requires the handling of singular and improper integrals. The proposed formulation comes from the HBEM variational base and its evaluation at internal points is straightforward without the application of any boundary integral, since the fundamental solution is the analytical one. This work belongs to a project that aims a computer code for large-scale problems (millions of degrees of freedom). At this stage, some numerical examples were analyzed to evaluate the applicability of the method expeditious its computational effort and convergence of the results for the variables involved in the method. It was developed by the algorithms implementation for potential and elasticity problems. In the case of two-dimensional were employed linear, quadratic and cubic elements and to the three-dimensional case were employed triangular, quadrilateral, linear and quadratic elements in both cases. The computational codes were always implemented focused on solving largescale problems. It is expected that in a final stage of the project with the incorporation procedure of the method "fast multipole", it can be more efficiently.

\section{Keywords}

Hybrid boundary elements; Variational methods; Numerical methods; Boundary elements; Large-scale problems. 


\section{Sumário}

1 Introdução 12

1.1 Colocação do problema 12

1.2 Objetivos 14

$\begin{array}{lll}1.3 & \text { Organização do texto } & 15\end{array}$

2 Considerações Teóricas Importantes $\quad 16$

2.1 Conceitos básicos da teoria de elasticidade linear 16

2.2 Conceitos básicos da teoria de potencial em regime permanente. 18

2.3 Discretização dos deslocamentos, das tensões e das forças de superfície 20

2.4 Aproximação da solução particular no contorno 22

2.5 Solução Fundamental. 23

3 Métodos de elementos de contorno $\quad 27$

3.1 O Método convencional dos elementos de contorno 27

3.2 O método híbrido dos elementos de contorno 33

3.3 O Método Híbrido Simplificado dos Elementos de Contorno 35

4 O Método Expedito dos Elementos de Contorno 38

4.1 Enunciados a partir do Princípio dos trabalhos virtuais 38

4.2 Aproximação dos deslocamentos e das forças de superfície no contorno 42

4.3 Expressões do Método Expedito dos Elementos de Contorno 43

4.4 Solução da equação matricial do problema e avaliação de resultados em pontos internos 48

5 Aplicações Numéricas $\quad 51$

5.1 Estudos de convergência em problemas de potencial 2D 51

5.2 Verificação da relação $\widetilde{\mathbf{H}} \mathbf{D}^{a} \approx \mathbf{U}^{* \mathrm{~T}} \mathbf{P}^{a}$ em problemas de potencial 3D 55

5.3 Problema de elasticidade 2D 58

5.4 Convergência para problemas de potencial 2D com fonte interna e condição de contorno mista

5.5 Convergência para problemas de elasticidade 2D com forças de massa e condição de contorno mista $\quad 70$

6 Conclusões e sugestões de trabalhos futuros $\quad \mathbf{7 6}$

6.1 Conclusões 76

$\begin{array}{lll}6.2 & \text { Sugestões de trabalhos futuros } & 78\end{array}$

$\begin{array}{ll}\text { Referências Bibliográficas } & 79\end{array}$

A Potencial de Hellinger-Reissner $\quad 83$

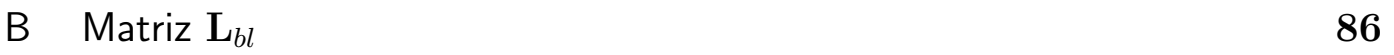




\section{Lista de figuras}

2.1 Corpo elástico equilibrado submetido à ação de forças externas, $b_{i}$ e $\bar{t}_{i}$, e deslocamentos prescritos $\bar{u}_{i}$.

2.2 Corpo homogêneo submetido a ações de fontes externas de energia, $Q$ e $\bar{q}_{n}$, e potencial prescrito $\bar{u}$.

4.1 Estrutura formada por seis nós e três elementos quadráticos para ilustração na construção das matrizes.

5.1 Discretização do contorno com uma malha de 124 nós para o domínio irregular. Para o estudo de convergência foram utilizados elementos lineares, quadráticos e cúbicos. A, B e C são pontos fonte.

5.2 Avaliação da convergência da equação (5-2), para os campos potenciais da Tabela 5.2, com a finalidade de estabelecer um ponto de erro como referência de convergência.

5.3 Normas de erro das matrizes, aproximadas, $\widetilde{\boldsymbol{H}}$ e $\boldsymbol{U}^{* T} \boldsymbol{L}^{T}$ de acordo com as equações equações (5-3) e (5-4), respectivamente, para elementos lineares, quadráticos e cúbicos.

5.4 Erros em escala logarítmica calculados com a norma euclidiana para testar a convergência dos métodos de elementos de contorno estudados. Resultados gerados por uma fonte potencial aplicada no ponto $C$ da Figura 5.1. Esquerda: Piores resultados. Direita: Melhores Resultados.

5.5 Hexaedro distorcido (à esquerda) e corpo multiplamente conectado feito de um cubo (à direita) para um estudo de convergência com elementos quadrilaterais lineares.

5.6 norma de erro do sistema de matrizes da equação (4-24), que aproxima as matrizes $\boldsymbol{H}$ e $\boldsymbol{U}^{*}$ no método expedito dos elementos de contorno, para diferentes discretizações da malha e o campo potencial aplicado aos corpos representados na figura 5.5 .

5.7 Discretização inicial do domínio irregular (62 nós) para o estudo de convergência e cálculo de tensões no domínio, utilizando elementos quadráticos. A e $B$ são pontos fonte.

5.8 Comparação do vetor $\boldsymbol{p}^{*}$, avaliada na equação (4-1) para forças nodais equivalente do contorno correspondente a um estado de tensão constante $\sigma_{x}$, com seus valores correspondentes do vetor $\widetilde{\boldsymbol{p}}^{*}$.

5.9 Erros das tensões, avaliados no segmento de reta (40 nós) entre os nós de coordenadas $(11,26)$ e $(20,7)$, ver Figura 5.7, para uma força horizontal aplicada no ponto $A$.

5.10 Comparação das tensões ao longo do segmento de reta entre os nós 18 e 34 que coincidem com o contorno $\Gamma$, ver figura 5.7, para uma força vertical aplicada no nó $A$.

5.11 Normas de erro, Equação (5-8), da equação matricial do método expedito, Equação (4-24), para elementos lineares, quadráticos e cúbicos. Os campos analíticos usados nas comparações correspondem a uma força horizontal (gráfico à esquerda) e uma vertical (gráfico à direita) aplicadas no ponto $B$ da Figura 5.7. 
5.12 Ilustração $3 D$ da solução particular $u^{p}$, gerado pela fonte interna da equação (5-9), que atua sobre o domínio irregular da figura 5.7.

5.13 Domínio irregular da Figura 5.7, submetido ao campo potencial dado pela equação (5-9) e ilustrado na Figura 5.12. O contorno correspondente aos nós $31-39$ está: isolado $q_{n}=0$ com potencial prescrito $u=0$ no resto do contorno (à esquerda), com potencial prescrito $u=0$ e isolado $q_{n}=0$ no resto do contorno (à direita).

5.14 Convergência dos potenciais u para diferentes malhas do domínio da Figura 5.13, grafico da esquerda. Usando elementos quadráticos.

5.15 Convergência dos gradientes $q_{n}$ para diferentes malhas do domínio da Figura 5.13, grafico da direita. Usando elementos quadráticos.

5.16 Malha inicial do domínio discretizado com 40 elementos quadráticos, utilizado para o cálculo de potencias e gradientes no contorno ao longo do segmento entre os nós 19 ao 25.

5.17 Convergência dos potenciais u para diferentes malhas do domínio da Figura 5.16, do segmento entre os nós $19-25$. Usando elementos quadráticos.

5.18 Convergência dos gradientes $q_{n}$ para diferentes malhas do domínio da Figura 5.16, do segmento entre os nós $19-25$. Usando elementos quadráticos. 69

5.19 Potenciais u para a malha $a_{5}$ da Tabela 5.10 do domínio da Figura 5.16, à esquerda. Resultados dos gradientes $q_{n}$ no segmento entre os nós $19-25$, à direita. Usando elementos quadráticos.

5.20 Domínio irregular da Figura 5.16, submetido a forças de massa dado pela equação (5-12). O contorno correspondente aos nós $19-25$ está: engastado $u=0$ com forças de superfície prescrito $t=0$ no resto do contorno (à esquerda), com forças de superfície prescritas $t=0$ e engastado $u=0$ no resto do contorno (à direita).

5.21 Convergência das Forças de superfície t na direção $x$ para as discretizações mostradas na Tabela 5.11 do domínio da Figura 5.16. Resultados do segmento entre os nós $19-25$. Usando elementos quadráticos.

5.22 Convergência das Forças de superfície t na direção y para as discretizações mostradas na Tabela 5.11 do domínio da Figura 5.16. Resultados do segmento entre os nós $19-25$. Usando elementos quadráticos.

5.23 Convergência dos deslocamentos u na direção $x$ para as discretizações mostradas na Tabela 5.11 do domínio da Figura 5.16. Resultados do segmento entre os nós $19-25$. Usando elementos quadráticos.

5.24 Convergência dos deslocamentos u na direção y para as discretizações mostradas na Tabela 5.11 do domínio da Figura 5.16. Resultados do segmento entre os nós $19-25$. Usando elementos quadráticos.

5.25 Deslocamentos u na direção $x$ e y para a malha ${ }_{5}$ da Tabela 5.11 do domínio da Figura 5.16, à esquerda. Resultados das forças de superfície $t$ na direção $x$ e y segmento entre os nós $19-25$, à direita. Usando elementos quadráticos. 75

A.1 Gráfico da energia interna de deformação. 


\section{Lista de tabelas}

4.1 Número de incógnitas e de soluções disponíveis (deslocamento de corpo rígido ou solução simples) e tipo de avaliação de coeficientes indefinidas (exato ou mínimos quadrados - m.q.) para cada fila da matriz, seja para problemas de potencial e elasticidade, 2D ou 3D.

5.1 Número total de nós utilizados nas diferentes malhas testadas para cada tipo de elemento (linear, quadrático ou cúbico).

5.2 Campos potenciais, obtidos analiticamente, aplicados para testar a convergência das equações matriciais do modelo numérico da Figura 5.1.

5.3 Dados das malhas discretizadas, para um estudo de convergência, correspondentes ao hexaedro distorcido (à esquerda da Figura 5.5).

5.4 Dados das malhas discretizadas, para um estudo de convergência, correspondentes ao corpo multiplamente conectado (à direita da Figura 5.5).

5.5 Campos potenciais, obtidos analiticamente, aplicados para testar a convergência das equações do modelo numérico da Figura 5.5.

5.6 Coordenadas $(x, y)$ dos nós localizados nos cantos do domínio irregular da Figura 5.\%.

5.7 Discretização das malhas utilizadas para o cálculo da convergência das tensões em pontos internos para o domínio da Figura 5.7.

5.8 Discretização das 6 malhas utilizadas para o teste de convergencia do sistema de equações matriciais do método expedito dos elementos de contorno para a estrutura da Figura 5.7.

5.9 Discretização das malhas utilizadas para o cálculo da convergência do problema de potencial em regime permanente e contorno misto, com elementos quadráticos, para o domínio da Figura 5.13.

5.10 Discretização das malhas utilizadas para o cálculo da convergência do problema de potencial em regime permanente e contorno misto, com elementos quadráticos, para o domínio da Figura 5.16.

5.11 Malhas utilizadas para o cálculo da convergência do problema de elasticidade linear e contorno misto, com elementos quadráticos, para o domínio da Figura 5.16. 
A mente que se abre a uma nova ideia jamais voltará ao seu tamanho original.

Albert Einstein. 